\title{
Evaluación funcional de deglución-aspiración en pacientes sometidos a laringectomía subtotal supracricoidea con cricohioidoepiglotopexia
}

\author{
José Francisco Gallegos-Hernández, María Elba Díaz-Guzmán, Pablo Antonio Pichardo-Romero, \\ Oscar Omar Mateos-Aguilar, José Alberto Abrego y Alma Lilia Ortiz-Maldonado \\ Hospital de Oncología, Centro Médico Nacional Siglo XXI, Instituto Mexicano del Seguro Social, Ciudad de México, México
}

\begin{abstract}
Resumen
Introducción: La laringectomía subtotal supracricoidea asociada con cricohioidoepiglotopexia es la técnica quirúrgica conservadora más eficiente para preservar las funciones laríngeas de los pacientes con carcinoma localmente avanzado de la laringe. La complicación más temida de esta intervención es la aspiración en el momento de la deglución y la neumonía secundaria; existen diversas formas de evaluar la aspiración y el grado de esta. Presentamos una novedosa forma de identificar incluso pequeñas cantidades de aspiración traqueobronquial. Método: Se incluyeron pacientes sometidos a laringectomía subtotal y cricohioidoepiglotopexia por cáncer laríngeo; todos evaluados con trago radiactivo posoperatorio. Con base en la sintomatología y resultado del gammagramma se decidió prolongar el tiempo de alimentación por sonda. Resultados: Se incluyeron 37 pacientes, cuatro habían recibido radioterapia; la tasa de aspiración fue de 29.7 \%; 50 \% de los pacientes que habían recibido radioterapia presentó aspiración y 18 \% de los pacientes con aspiración requirió prolongación del tiempo de alimentación por sonda nasogástrica; ninguno necesitó laringectomía total por aspiración que no permitiera la deglución. Conclusiones: La evaluación posoperatoria de pacientes sometidos a laringectomía subtotal con trago radiactivo permite identificar líquido aspirado al árbol bronquial, incluso en cantidades mínimas, y planear el momento para iniciar la deglución.
\end{abstract}

PALABRAS CLAVE: Cricohioidoepiglotopexia. Laringectomía. Laringectomía subtotal. Laringectomía parcial. Deglución. Cáncer de laringe.

\begin{abstract}
Introduction: Supracricoid subtotal laryngectomy associated with cricohyoidoepiglottopexy is the most efficient conservative surgical technique to preserve laryngeal functions in patients with locally advanced carcinoma of the larynx. The most feared complication of this intervention is aspiration at the moment of swallowing and secondary pneumonia; there are several ways to evaluate aspiration and its degree. We present a novel form to identify even small amounts of tracheobronchial aspiration. Method: Patients undergoing subtotal laryngectomy and cricohyoidoepiglottopexy due to laryngeal cancer were included; all of them were postoperatively assessed with radioactive-swallow. Based on the symptoms and the scintigraphy result, prolonging the feeding time by nasogastric tube was decided. Results: Thirty-seven patients were included; 4 of them had received radiotherapy; aspiration rate was $29.7 \% ; 50 \%$ of patients who had received radiation had aspiration; $18 \%$ of subjects with tracheobronchial aspiration required prolongation of feeding time by nasogastric tube, none required total laryngectomy for aspiration that hindered swallowing. Conclusions: Postoperative evaluation of patients undergoing subtotal laryngectomy with radioactive-swallow, allows to identify even minimal amounts of fluid aspirated into the bronchial tree and to plan the moment for swallowing to be started.
\end{abstract}

KEY WORDS: Cricohyoidoepiglottopexy. Laryngectomy. Subtotal laryngectomy. Partial laryngectomy. Swallowing. Laryngeal cancer.

Correspondencia:

José Francisco Gallegos-Hernández

E-mail: jfgalh61@gmail.com
Fecha de recepción: 01-12-2017

Fecha de aceptación: 10-09-2018

DOI: 10.24875/GMM.18003936
Gac Med Mex. 2018;154:645-648

Disponible en PubMed

www.gacetamedicademexico.com 


\section{Introducción}

La laringectomía parcial denominada subtotal-supracricoidea (LSSC) fue introducida en Europa entre las décadas de 1950 y 1970;1,2 fue diseñada como un paso previo a la laringectomía total para preservar la ventilación por vía natural y la fonación (que no es posible con la laringectomía total) y permitir al paciente deglutir sin aspiración traqueobronquial, además de ofrecer adecuado control oncológico. ${ }^{1}$

Actualmente la LSSC tiene un papel importante en el tratamiento de pacientes con carcinoma epidermoide de laringe en etapas localmente avanzadas que no son candidatos a tratamiento conservador no quirúrgico ya sea con radioterapia sola o quimiorradioterapia concomitante,$^{3}$ así como en algunos pacientes con recurrencia posterior a radioterapia. Sin embargo, este procedimiento no está exento de complicaciones, la más frecuente es la aspiración bronquial al retirar la cánula de traqueotomía e iniciar la deglución. La aspiración importante es una indicación para mantener la cánula de traqueotomía para evitar el riesgo de neumonía por aspiración o de continuar por más tiempo la alimentación por sonda nasogástrica; la broncoaspiración persistente a pesar de realizar rehabilitación de la deglución es una indicación absoluta para convertir el procedimiento conservador en laringectomía total. ${ }^{4,5}$

La broncoaspiración debe diagnosticarse oportunamente, ya que puede no ser clínicamente evidente y hacerse presente tardíamente con focos neumónicos, lo que compromete seriamente la evolución posoperatoria, deteriora el estado de salud del paciente y pone en peligro su vida.

La aspiración en pacientes sometidos a laringectomía subtotal es importante: 30 a $40 \%$ puede presentarla en diversos grados; es más frecuente en pacientes mayores de 65 años, en quienes la cirugía se efectúa en forma de rescate posterior a radioterapia y en pacientes en quienes se ha comprometido la función de uno de los cartílagos aritenoides, ya sea por resección o lesión quirogénica del nervio laríngeo inferior. ${ }^{4,6,7}$

Las formas habituales para identificar si el paciente tiene aspiración bronquial al momento de la deglución son la evaluación clínica (interrogatorio dirigido a conocer si existe tos con la deglución), la videoendoscopia y la videofluorescencia. Sin embargo, con estos métodos, cantidades leves a moderadas de líquido aspirado pueden pasar inadvertidas y repercutir tardíamente en el estado ventilatorio del paciente.
El objetivo del presente estudio es describir una novedosa técnica para evaluar la aspiración en pacientes sometidos a laringectomía subtotal supracricoidea, que permite identificar cantidades mínimas de aspiración del líquido al árbol bronquial: el trago radiactivo, que permite planear con mayor objetividad el manejo de la traqueotomía.

\section{Método}

Se evaluaron pacientes con carcinoma epidermoide de laringe que en forma consecutiva fueron sometidos a conservación quirúrgica de la laringe con LSSC y reconstrucción con cricohioidoepiglotopexia (CHEP).

El retiro de la sonda nasogástrica y la decanulación se efectuó en forma consecutiva y según la evaluación clínica. Una semana posterior a la decanulación se realizó trago con material radiactivo y gammagrafía para detectar líquido aspirado y decidir si se requería recolocación de sonda de alimentación y rehabilitación; de no haber aspiración importante se decidía continuar sin sonda y con ejercicios de rehabilitación.

La técnica quirúrgica fue similar en todos los pacientes: se conservaron ambos cartílagos aritenoides y las indicaciones para efectuar el procedimiento fueron las habitualmente reportadas; en todos los pacientes se tomaron medidas durante el transoperatorio para disminuir al mínimo la aspiración (se evitó colocar puntos de sutura endolaríngeos, puntos para fijación de cartílagos corniculados y cerrar la brecha faríngea para no favorecer la rotación del seno piriforme y con ello la aspiración).

Para la técnica del trago radiactivo se siguió protocolo de adquisición; se administró $1 \mathrm{mCi}$ en $1 \mathrm{~mL}$ de volumen por vía oral de ${ }^{99 \mathrm{~m}} \mathrm{Tc}$ sulfuro coloidal, se utilizó una gammacámara Phillips Precedence T16 y un colimador de baja energía para propósitos generales, con ventana de $20 \%$, centrada en fototópico de $140 \mathrm{KeV}$.

Se realizó estudio dinámico $64 \times 564$ e imágenes estáticas de $256 \times 256$. Las imágenes tomadas en la fase dinámica fueron 30 de 2 segundos y en la fase estática una imágen inmediata de 2 a 3 minutos de duración y otra a las dos horas, de 5 minutos de duración. Las proyecciones fueron anterior y posterior y el campo de visión abarcó de la región maxilar hasta el epigastrio.

Con el paciente de pie frente al detector de la gammacámara se administró $1 \mathrm{~mL}$ del radiofármaco por vía oral, iniciando el estudio dinámico por 60 segundos, posteriormente se le hizo deglutir $5 \mathrm{~mL}$ de 
agua y se efectuaron las imágenes estáticas inmediatas y a las dos horas las tardías.

Se efectuó valoración cualitativa (visual) para identificar paso del radiofármaco a la vía aérea y valoración cuantitativa trazando áreas de interés (Figura 1).

Independientemente del estado clínico, los pacientes que presentaban aspiración se sometieron a rehabilitación foniátrica y de deglución en forma intensiva si no tenían síntomas o a colocación de cánula de traqueotomía y sonda nasogástrica, además de rehabilitación, si había evidencia clínica de aspiración.

\section{Resultados}

Se incluyeron 37 pacientes sometidos a LSSC y CHEP por carcinoma epidermoide de laringe; en 33, el procedimiento quirúrgico fue efectuado en forma primaria, en tres como rescate posterior a radioterapia y en uno posterior a biorradioterapia.

El trago radiactivo mostró aspiración bronquial en $11 / 37$ pacientes $(29.7 \%$ ) y no mostró material radiactivo en la región traqueobronquial en $70.3 \%$. De ellos, 8/33 de los que no habían tenido tratamiento previo (24\%) presentaron aspiración y 2/4 (50\%) de los que habían recibido tratamiento (radioterapia) presentaron aspiración bronquial. El paciente con antecedente de biorradioterapia fue el que presentó mayor cantidad de aspiración y requirió más tiempo de sonda nasogástrica.

Los pacientes sometidos al procedimiento en forma inicial tuvieron una tasa de aspiración de $27.7 \%$, en los operados en forma de rescate la tasa de aspiración fue de $50 \%$.

De los pacientes que mostraron aspiración (11), tres (27 \%) tuvieron sintomatología clínica importante que requirió la sonda de alimentación: en dos por 10 días más y en otro por tres semanas más, después de las cuales el trago mostró mínima aspiración y la deglución era tolerada adecuadamente.

Los pacientes que en el estudio gammagráfico mostraron aspiración limitada a la tráquea o mínima aspiración a uno de los bronquios toleraron la decanulación y se reintegraron a una dieta normal en promedio dos semanas después de la decanulación y retiro de la sonda nasogástrica.

Los 37 pacientes fueron finalmente decanulados y se les retiró la sonda nasogástrica; ninguno mostró focos neumónicos durante su tratamiento ni requirió conversión de la LSSC a laringectomía total; el tiempo medio de seguimiento es de dos años y ninguno ha presentado complicaciones por aspiración.

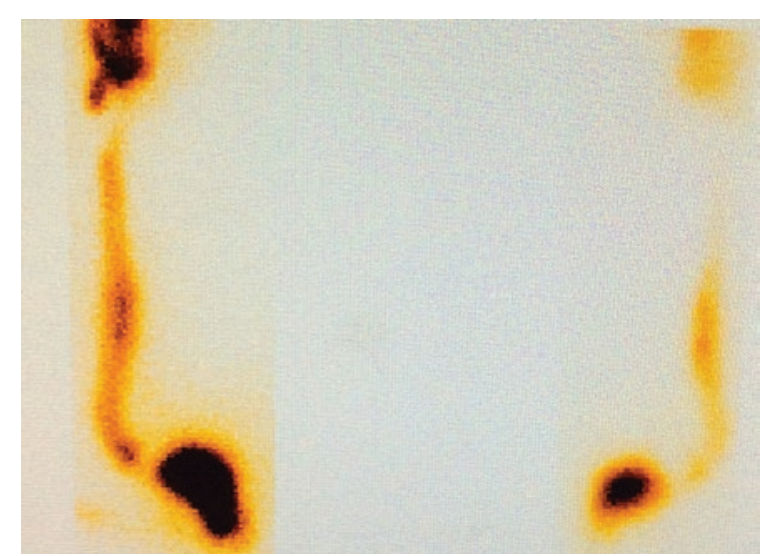

Figura 1. Trago radiactivo que muestra adecuado paso del radiofármaco hacia el esófago sin aspiración bronquial, lo que ocurrió en $70.3 \%$ de los pacientes; el retiro de la sonda de gastrostomía y de la cánula de gastrostomía puede hacerse sin riesgo de broncoaspiración.

\section{Discusión}

La LSSC con CHEP es una adecuada alternativa para conservar las funciones laringeas en pacientes con cáncer de laringe localmente avanzado no candidatos a radioterapia ni a quimiorradioterapia o como procedimiento de rescate por recurrencia a radioterapia o quimiorradioterapia., ${ }^{3,8,9}$ Permite lograr control oncológico adecuado y funcionalidad aceptable de la laringe; la complicación más importante es la aspiración de los alimentos, que se ha asociado con edad avanzada, ${ }^{10}$ resección de un aritenoides y formación de granulomas endolaríngeos. Una técnica quirúrgica adecuada permite disminuir al mínimo la tasa de aspiración, la cual en muchas ocasiones no es clínicamente evidente. ${ }^{11}$

La evaluación posoperatoria de la deglución es fundamental para identificar aspiración traqueobronquial e iniciar medidas para disminuir la posibilidad de complicaciones por neumonía; ${ }^{12}$ el trago radiactivo con gammagrafía permite identificar la cantidad de aspiración y decidir si el paciente es candidato a ejercicios de rehabilitación, recolocación de la sonda nasogástrica 0 de la cánula de traqueotomía y eventualmente la conversión del procedimiento a laringectomía total.

En la presente serie se identificó aspiración en casi $30 \%$ de los pacientes, en $82 \%$ de ellos la cantidad de líquido aspirado evaluado con la gammagrafía posterior a trago radicativo estuvo limitado a la tráquea y a uno solo de los bronquios en cantidad mínima, lo que permitió el reinicio de la deglución sin riesgo de aspiración mayor; estos pacientes no requirieron colocación de cánula de traqueotomía ni de sonda nasogástrica y la aspiración mínima fue resuelta con 
terapia posoperatoria para la deglución; $18 \%$ de los pacientes necesitó mayor tiempo de alimentación por sonda nasogástrica y mayor tiempo de traqueotomía. Ninguno requirió conversión a laringectomía total ni presentó complicaciones neumónicas durante su seguimiento; todos se reintegraron a una dieta normal.

En la presente serie, aunque el número de pacientes en los que se efectuó la LSTSC como rescate (recaída posterior a radioterapia) fue escaso, en ellos la tasa de aspiración fue mayor (50\%), probablemente asociada con disfunción de la articulación cricoaritenoidea debida a morbilidad posradioterapia.

La no evaluación de la deglución en el posoperatorio de cirugía conservadora de laringe hace que el retiro de la sonda nasogástrica se decida con base en la sintomatología clínica, ${ }^{13}$ lo que favorece el mayor tiempo de nutrición por la sonda y el retraso en la rehabilitación. ${ }^{7,13}$

El trago radiactivo, empleado frecuentemente en los niños para evaluación de broncoaspiración por diversas causas, ${ }^{14}$ es útil para identificar la más mínima cantidad de líquido aspirado al árbol traqueobronquial; con base en el resultado se decide el momento ideal del retiro de la sonda de traqueotomía y el reinicio de la deglución, con lo que se minimiza la posibilidad de compliaciones respiratorias.

\section{Bibliografía}

1. Majer EH, Rieder W. Modification of laryngectomy with preservation of the air passages. Arch Ohren Nasen Kehlkopfheilkd. 1958;173:442-446.

2. Piquet JJ. Functional laryngectomy (cricohyoidopexy). Clin Otolaryngol Allied Sci. 1976;1:7-16.

3. Gallegos-Hernández JF, Cruz-Esquivel I, Ortiz-Maldonado AL, Minauro-Muñoz GG, Arias-Ceballos H, Pichardo-Romero P. Laryngeal conservative surgery in patients candidates for combined treatment with chemoradiotherapy. Cir Cir. 2016;84:96-101.

4. Leone CA, Capasso P, Topazio D, Russo G. Supracricoid laryngectomy for recurrent laryngeal cancer after chemoradiotherapy: a systematic review and meta-analysis. Acta Otorhinolaryngol Ital. 2016;36:439-449.

5. Zietek E, Jaworowska E, Tarnowoska C, Jach K Functional results after supracricoid reconstructive laryngectomy with cricohyoidopexy (chp) and cricohioidoepiglotopexy (chep). Otolaryngol Pol 1992;46:457-462.

6. Allegra E, Franco T, Traspasso S, Domanico R, La-Boria A, Garozzo A. Modified supracricoid laryngectomy: oncological and functional outcomes in the elderly. 2012;7:475-480.

7. Clayburgh DR, Graville DJ, Palmer AD, Schindler JS. Factors associated with supracricoid laryngectomy functional outcomes. Head Neck. 2013;35:1397-1403.

8. Manelli G, Lazio M, Luparello P, Gallo O. Conservative treatment for advanced T3-4 laryngeal cancer: meta-analysis of key oncological outcomes. Eur Arch Otorhinolaryngol. 2018;275:27-38.

9. Luna-Ortiz K, Pashe P, Tamez-Velarde M, Villavicencio-Valencia V. Spracricoid partial laryngectomy with cricohyoidoepiglottopexy in patients with radiation therapy failure. World J Surg Oncol. 2009;7:101.

10. Laccourreye $O$, Brasnu D, Périé D, Muscatello L, Ménard M, Weinstein G. Supracricoid partial laryngectomies in the elderly: mortality, complications and functional outcome. Laryngoscope. 1998;108:237-242.

11. Topaloglu I, Köprücü G, Bal M. Analysis of swallowing function alter supracricoid laryngectomy with cricohyoidopexy. Otoalryngol Head Neck Surg. 2012;146:412-418.

12. Yücetürk AV, Tarhan S, Günhan K, Pabușçu Y. Videofluorscopic evaluation of the swallowing function after supracricoid laryngectomy. Eur Arch Otorhinolaryngol. 2005;262:198-203.

13. De Vincentiis MI, Calcagno P, Di-Cello P, Mastronicola R, Simnelli M, Ruoppolo G, et al. Transit time of swallowing after subtotal laryngectomy. Rev Laryngol Otol Rhinol (Bord). 2004;125:223-227.

14. Heyman $\mathrm{S}$. The radionuclide salivagram for detectin the pulmonary aspiration of saliva in an infant. Pediatr Radiol. 1989;19:208-209. 